\title{
Mechanism of Anionic Polymerization of (Meth)acrylates in the Presence of Aluminium Alkyls IV. Formation of a Co-ordinative Polymer Network via the Living Aluminate End Group*
}

\author{
Helmut SchlaAd and Axel H. E. MülleR ${ }^{\dagger}$
}

Institut für Physikalische Chemie, Universität Mainz, Welder-Weg 15, D-55099 Mainz, Germany

(Received March 27, 1996)

\begin{abstract}
The polymerization of methyl methacrylate in the presence of aluminium alkyls in toluene deviates from conventional kinetics. This results predominantly from the formation and precipitation of a co-ordinative polymer gel or network. Due to the lower reactivity and accessibility of the living chains in the gel, they are regarded as 'dormant' and thus the concentration of active species decreases during polymerization. The network formation occurs via co-ordination of the living aluminate chain end group with in-chain ester carbonyl groups. Part of the chains are deactivated by a termination process but they are free of cyclic $\beta$-ketoesters which would result from the common 'back-biting' reaction.
\end{abstract}

KEY WORDS Anionic Polymerization / Poly(methyl methacrylate) / Aluminium Alkyls / Co-ordinative Polymer Network /

In recent publications, ${ }^{1-3}$ we described the anionic polymerization of methyl methacrylate in the presence of aluminium alkyls in toluene at $-78^{\circ} \mathrm{C}$ as deviating from conventional first-order kinetics. The first-order time-conversion plots are kinked at low monomer conversions, i.e., the rate of polymerization strongly decreases after an initially fast reaction. Since the polymers are free of cyclic $\beta$-ketoesters, the decrease in rate can hardly be explained by 'back-biting' termination. Another approach to explain the kinetic data assumes an intra- or intermolecular co-ordination of in-chain ester carbonyl groups to the aluminate end group during the early monomer addition steps, i.e., the existence of at least two active species. ${ }^{2,3}$

Fontanille et $a l .{ }^{4}$ also found kinked time-conversion plots in the anionic polymerization of 2-vinyl pyridine which has an isoelectronic structure to methyl methacrylate - in the presence of triethylaluminium. There, the decrease of the rate of polymerization coincides with the precipitation of a solid which is supposed to be a coordinative network of living polymer chains. A network of poly(methyl methacrylate) instead would be isorefractive with toluene and thus difficult to be observed in that solvent. Nevertheless, Hatada et al. ${ }^{5}$ found 'gel-like particles' in the presence of triethylaluminium at $-78^{\circ} \mathrm{C}$, Haddleton et $a l^{6}$ reported 'polymer gel formation' with triisobutylaluminium at $0^{\circ} \mathrm{C}$, and so did we with triethylaluminium at temperatures above $-65^{\circ} \mathrm{C} .^{3}$ However, all of these reports are restricted to the statement that a polymer gel precipitates during polymerization but do not give further information. In the present work, we propose a tentative structure of the polymer gel based on spectroscopic studies. Additionally, we present some details of the properties of the polymer gel - especially its living character towards monomer addition - and its role in polymerization kinetics.

\section{EXPERIMENTAL}

\section{Reagents}

Methyl methacrylate (MMA, Röhm $\mathrm{GmbH}$ ) was fractionated from $\mathrm{CaH}_{2}$ over a 1-m column filled with Sulzer packing at 45 mbar. After degassing, the distillate was stirred over $\mathrm{CaH}_{2}$ and distilled in high vacuum. Toluene and tetrahydrofuran (BASF AG) were fractionated over a 1.5-m column, twice stirred over sodium/ potassium alloy, degassed, and distilled in high vacuum. tert-Butyl lithium (tBuLi, $1.7 \mathrm{M}$ in pentane, Aldrich) was titrated and used without further purification, triethylaluminium ( $\mathrm{AlEt}_{3}, 25 \mathrm{wt} \%$ in toluene, Aldrich) was used as received. Benzoyl chloride (Aldrich) was stirred over cross-linked poly(4-vinyl pyridine) (Reillex ${ }^{\mathrm{TM}} 402$, Aldrich) to remove acid impurities, degassed, and distilled in vacuum.

\section{Polymer Gel Preparation}

The polymerizations were carried out in toluene at room temperature under nitrogen atmosphere. Typical concentrations of the reagents were $[\mathrm{tBuLi}]_{0}=(0.4$ $\cdots 1.7) \times 10^{-2} \mathrm{moll}^{-1}, \quad\left[\mathrm{AlEt}_{3}\right]_{0}=(1.5 \cdots 3.4) \times 10^{-2}$ moll $1^{-1}$, and $[\mathrm{MMA}]_{0}=0.2 \mathrm{moll}^{-1}$. The polymer gel was separated from the supernatant reaction solution by filtration, washed with toluene, and dried in high vacuum. Both polymer fractions were quenched with methanol or benzoyl chloride respectively. The monomer conversion, $x_{\mathrm{p}}$, was determined from the reaction solution with GC using octane as internal standard and the yield of the polymer gel, $y_{\text {gel }}$, was determined by gravimetric analysis. From these data, the weight fractions of the polymer in solution, $w_{\text {sol }}$, and in gel, $w_{\text {gel }}$, were calculated: $w_{\mathrm{gel}}=1-w_{\mathrm{sol}}=y_{\mathrm{gel}} /\left(x_{\mathrm{p}} \times[\mathrm{MMA}]_{0}\right)$. The mole fractions, $x_{\text {sol }}$ and $x_{\text {gel }}$, were determined by dividing the weight fractions by the corresponding number average molecular weight.

\footnotetext{
* Part III: H. Schlaad, A. H. E. Müller, H. Kolshorn, and R. P. Krüger, Polym. Bull., 35, 169 (1995).

† To whom correspondence should be addressed.
} 


\section{Polymerization}

In a typical experiment, a solution of $260 \mathrm{mg}$ MMA in $1 \mathrm{ml}$ THF was added within a few seconds to $c a .40$ $\mathrm{mg}$ of a living polymer network (dried in high vacuum) at room temperature under nitrogen atmosphere. The reaction was quenched with methanol after five minutes.

\section{$G P C$}

GPC was performed using tetrahydrofuran (THF) as eluent at a flow rate of $1 \mathrm{ml} \mathrm{min}^{-1}$. Detectors: $2 \times$ JASCO-UVIDEC 100 III with variable wavelength and Bischoff RI detector 8110 , column set: $1 \times 5 \mu \mathrm{m} / 100$ $\AA / 60 \mathrm{~cm}, 1 \times 5 \mu \mathrm{m} / 10^{2}-10^{5} \AA / 60 \mathrm{~cm} /$ PSS gel. PMMA standards were used for calibration. If necessary, the MWD's were corrected according to the relative response factor, $f: R I_{\text {korr }}=R I / f ; f=1-\alpha /(M-\beta)$ with $\alpha=107$, $\beta=34$. The coefficients $\alpha$ and $\beta$ were determined on the basis of the relative refractive index increments of $\mathrm{H}(\mathrm{MMA})_{n} \mathrm{H}, f=-0.54(n=1), 0.34$ (2), 0.58 (3), 0.74 (4), and $\mathrm{tBu}(\mathrm{MMA})_{n} \mathrm{H}, f=0.06(n=1), 0.59$ (2).

\section{CP-MAS Solid-State NMR}

Dipolar broadband decoupled ${ }^{13} \mathrm{C}$ NMR spectra were recorded on a Bruker MSL-300 NMR spectrometer equipped with a standard Bruker MAS $7 \mathrm{~mm}$ probe head. The samples were spun at frequencies of $3 \mathrm{kHz}$. The $90^{\circ}$-pulse lengths were in the range of $3.5-4.0 \mu \mathrm{s}$ and 3000 scans were accumulated with a repetition time of $2 \mathrm{~s}$ for each spectrum. ${ }^{27} \mathrm{Al}$ NMR spectra were recorded on a Bruker ASX-500 spectrometer equipped with a standard Bruker MAS $4 \mathrm{~mm}$ probe head. The samples were spun at $14 \mathrm{kHz}$. The $90^{\circ}$-pulse lengths were $1 \mu \mathrm{s}$. All chemical shifts are given relative to tetramethylsilane and aqueous aluminium chloride solution, respectively, as external standard.

\section{RESULTS AND DISCUSSION}

\section{Isolation and Characterization of the Precipitate}

Above $-65^{\circ} \mathrm{C}$, a colorless gel sticks at the glass wall of the reaction vessel when polymerizing methyl methacrylate with tert-butyl lithium/triethylaluminium in toluene, i.e., the reaction solution becomes heterogeneous. ${ }^{3}$ The gel immediately dissolves in polar solvents like tetrahydrofuran and, after quenching the reaction with methanol, in toluene. NMR and GPC analysis prove that the gel consists of poly(methyl methacrylate) with number-average molecular weights of usually less than 10.000 ( $c f$. ., Figure 1). Since a linear polymer with such a low molecular weight is easily soluble in toluene, the insolubility must be related to an aggregation process.

In order to obtain further information about the precipitated poly(methyl methacrylate), the above described polymerization was repeated at room temperature and the gel was separated from the supernatant reaction solution. In that case, the weight fraction of precipitated polymer is $c a$. $70 \%$ and GPC analysis (Figure 1) shows that it has a broad molecular weight distribution $\left(M_{w} / M_{n}=1.8\right)$ including even the lowest oligomers.

In the ${ }^{13} \mathrm{C} \mathrm{CP} / \mathrm{MAS}$ solid-state NMR spectrum of the unquenched polymer gel the expected signals of poly(methyl methacrylate) are detected: $\delta / \mathrm{ppm}=19\left(-\mathrm{CH}_{3}\right)$,

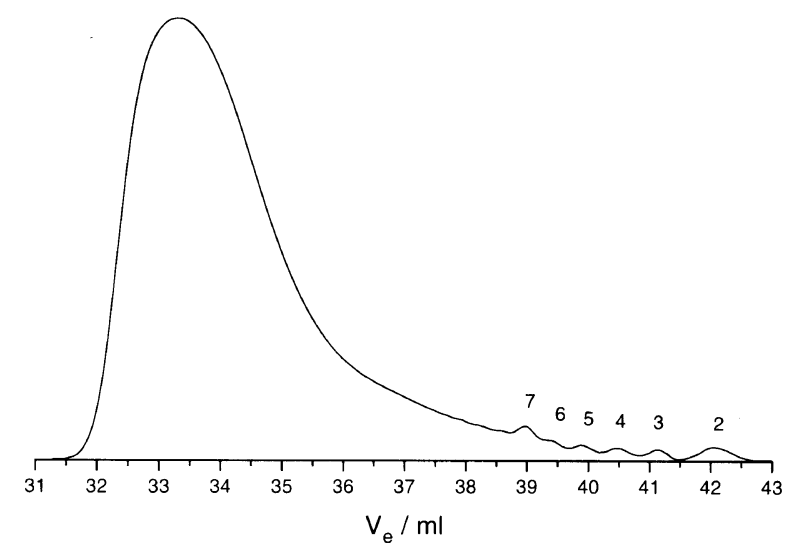

Figure 1. GPC eluogram of the precipitated poly(methyl methacrylate) after quenching with methanol, $M_{n}=3040, M_{w} / M_{n}=1.8$. The numbers, $n$, correspond to the oligomers $\mathrm{tBu}(\mathrm{MMA})_{n} \mathrm{H}$.

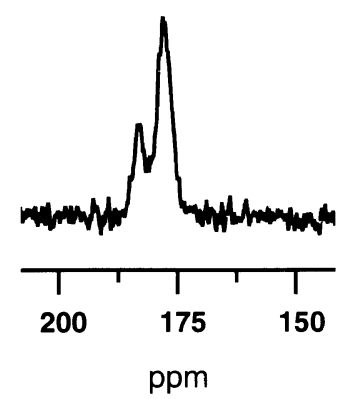

Figure 2. Ester carbonyl region of the ${ }^{13} \mathrm{C} \mathrm{CP}-\mathrm{MAS}$ solid-state NMR spectrum of poly(methyl methacrylate) gel prepared with $\mathrm{tBuLi}^{\mathrm{A}} \mathrm{AlEt}$ in toluene at room temperature.

$45\left(-\mathrm{CH}_{2}-\right), 53\left(-\mathrm{OCH}_{3}\right), 178(\mathrm{C}=\mathrm{O})$. Additionally, we find two signals of ethyl groups bound to aluminium $(\delta=2$ and $12 \mathrm{ppm})$ and one of a second ester carbonyl group ( $\delta=184 \mathrm{ppm}$, see Figure 2 ). The latter signal is assigned to ester carbonyl groups co-ordinated to excess triethylaluminium or to the aluminate end group. Similarly, the ${ }^{13} \mathrm{C}$ NMR signal of the penultimate ester carbonyl group of the di-tert-butyl $\alpha$-lithioglutarate triethylaluminium complex shifts from $\delta=176$ to $183 \mathrm{ppm}^{3}$ and the $\mathrm{C}(\mathrm{O})$ signal of methyl pivalate shifts from $\delta=178$ to $182 \mathrm{ppm}$ in the presence of equimolar amounts of triisobutylaluminium in toluene. ${ }^{7}$ For aluminium alkyls it is known that they only form $1: 1$ complexes with esters $^{7}$ or other electron-donating agents (e.g., ethers or amines) ${ }^{8}$ i.e., an aggregation of polymer chains via the excess triethylaluminium is rather questionable. In fact, it is not possible to precipitate a quenched poly(methyl methacrylate) from toluene solution by adding triethylaluminium. We assume that this NMR shift of ester carbonyl groups predominantly results from their co-ordination to the aluminate end group. Since the NMR signals of the quaternary carbon atoms of the ester enol aluminate group were considerably broad even for the living dimer in toluene, ${ }^{3}$ we can hardly expect any signal for the living chain end in the solid-state NMR spectrum. Nevertheless, the polymer chains incorporated in the precipitate have living character as indicated by their ability to further add monomer (see below).

In the corresponding ${ }^{27} \mathrm{Al}$ NMR spectrum (see Figure 3 ), a predominant signal at $\delta \approx 14 \mathrm{ppm}$ was detected, i.e., 
the aluminium of the end group is six-fold co-ordinated in the precipitate. If excess triethylaluminium was responsible for aggregation we would expect a maximum co-ordination number of five corresponding to $\delta \approx 120$ ppm. ${ }^{9}$

Based on co-ordination numbers of four and five in unimeric and dimeric ester enol aluminates, respectively, we suppose that the increase of the co-ordination number is achieved by the co-ordination of two in-chain ester groups to unimeric aluminate end groups or of one ester group to dimeric chain ends. Regarding a polymer chain

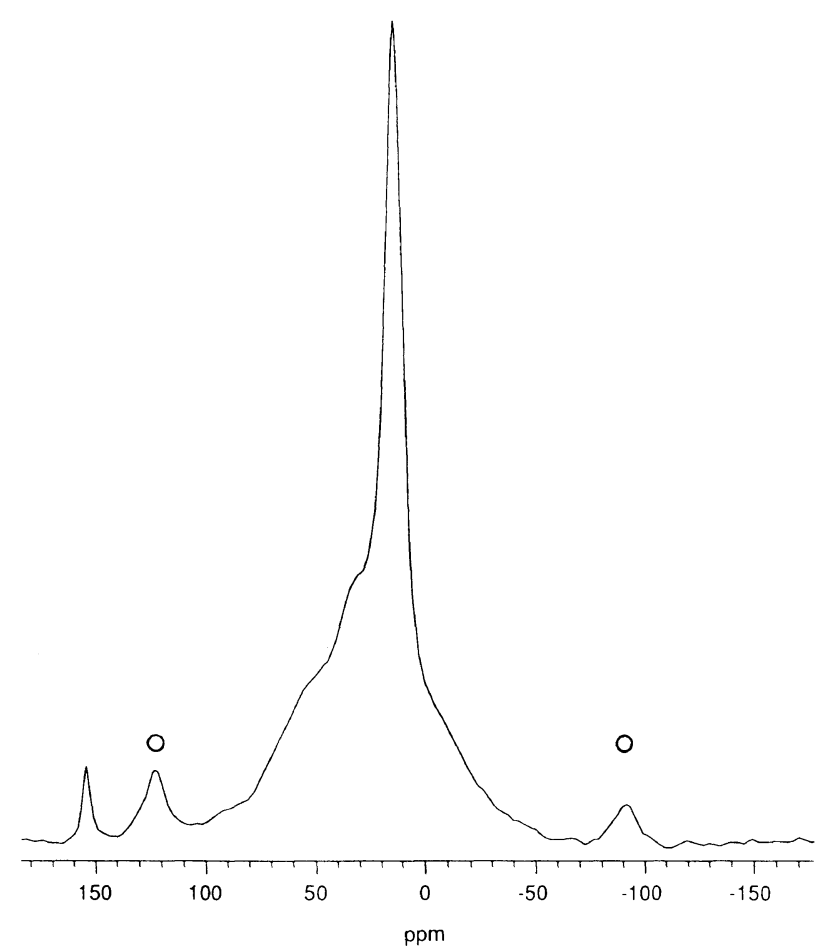

Figure 3. ${ }^{27} \mathrm{Al}$ CP-MAS solid-state NMR spectrum of poly(methyl methacrylate) gel prepared with $\mathrm{tBuLi} / \mathrm{AlEt}_{3}$ in toluene at room temperature. The signal at $\delta=154 \mathrm{ppm}$ is assigned to result from residual triethylaluminium, ${ }^{9} \mathrm{O}=$ spinning sidebands. as a mono- or a bi-dentate ligand, we would expect a co-ordinative polymer network to be formed (see Figure 4). From entropy considerations, the structure given for dimeric chain ends is less probable.

\section{Living Character of the Co-ordinated Polymer Network}

In order to check the living character of such a co-ordinated polymer network, methyl methacrylate in tetrahydrofuran was added to the isolated gel at room temperature (under these conditions, the network dissolves readily). The GPC eluogram of the product (Figure 5) shows a considerable chain extension in addition to some residual precursor polymer, i.e., part of the polymer chains in the network were not living. From the expected and the experimentally determined average molecular weight of the 'block' polymer, the mole fraction of living chains in the network was calculated to be at least $60 \%$.

In order to distinguish the living from the terminated polymer chains, the polymer network was quenched with benzoyl chloride. The UV absorption of the phenyl group $\left(\lambda_{\max } \approx 260 \mathrm{~nm}\right)$ is proportional to the number of active chains. Thus, the RI and the UV signals in GPC analysis are proportional to mass and frequency, respectively. In order to compare both signals, the RI signal was transformed into a frequency-proportional signal by dividing each GPC slice with the corresponding molecular weight. The ratio $\mathrm{UV} /(\mathrm{RI} / \mathrm{M})$ (see Figure 6) indicates that the mole fraction of polymer chains with UV label is significantly lower in the oligomer region. The resolved oligomer peaks are assigned as linear $\mathrm{tBu}(\mathrm{MMA})_{n} \mathrm{H}$ and no signals due to cyclic $\beta$-ketoester end groups were found, i.e., termination via the usual 'backbiting' reactin did not occur. It appears that the termination of the living chains is, however, closely connected to their incorporation into the network but the mechanism of this termination reaction is not understood yet and will be subject of further studies.
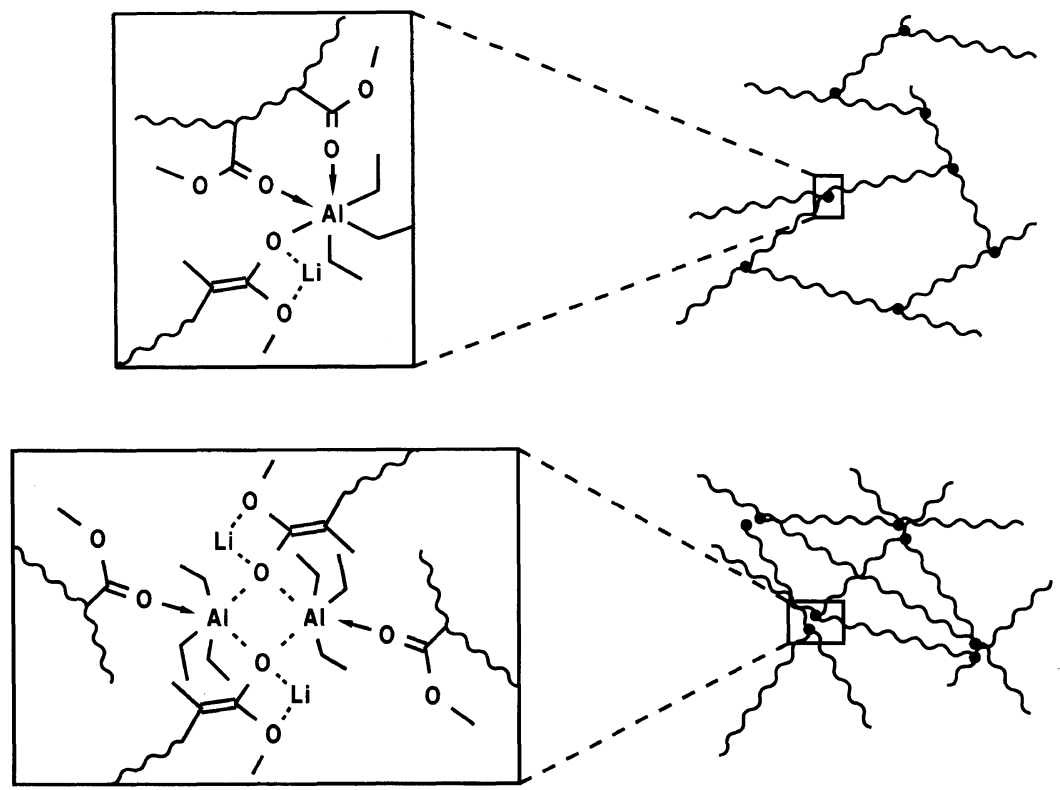

Figure 4. Tentative structures resulting from co-ordination of polymer chains to an unimeric or dimeric aluminate end group (O approximate location of the lithium is based on ab-initio calculations on the methyl $\alpha$-lithioisobutyrate triethylaluminium ate complex. 


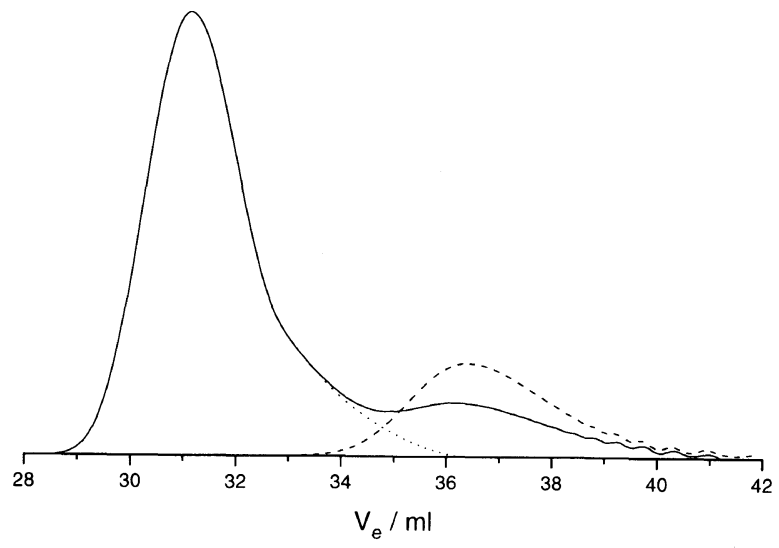

Figure 5. GPC eluogram of a 'block' polymer (,$- M_{n}=16170$, $M_{w} / M_{n}=1.8$ without the precursor polymer) obtained by the polymerization of methyl methacrylate initiated by a co-ordinated polymer network (---, $\left.M_{n}=1260, M_{w} / M_{n}=1.3\right)$ in tetrahydrofuran at room temperature, monomer conversion $x_{\mathrm{p}}=0.42$. The areas are normalized to the corresponding polymer mass.

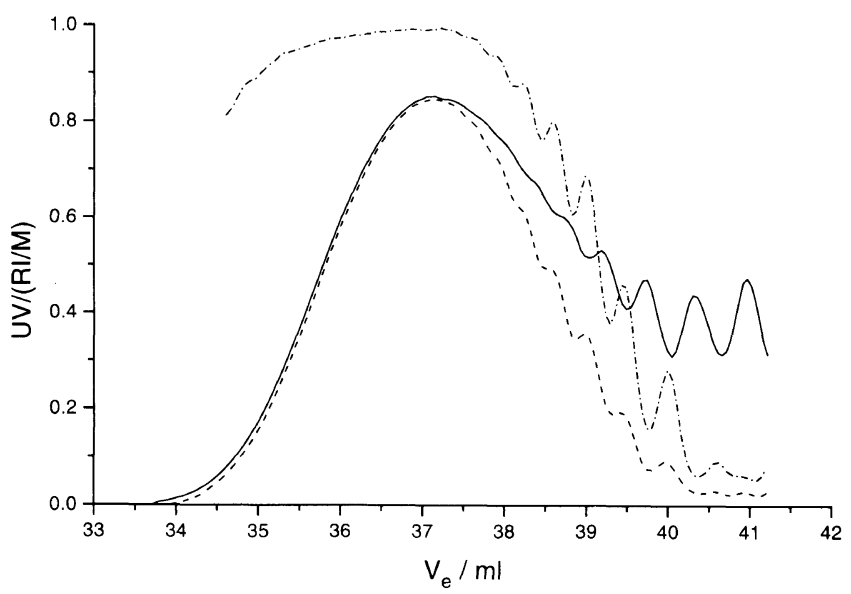

Figure 6. Frequency distribution of a co-ordinative network ( $c f$., Figure 5) quenched with benzoyl chloride ([benzoyl chloride]/[tBu$\mathrm{Li}] \approx 5)$. - , RI/M; -.-, UV $(\lambda=260 \mathrm{~nm}) ; \cdot \cdots \cdot-\cdot, \mathrm{UV} /(\mathrm{RI} / \mathrm{M})$. The RI signal is corrected with respect to the relative RI response factor of the polymer.

Effect of the Co-ordinated Polymer Network on Polymerization Kinetics

For an 'ideal' polymerization, the rate of polymerization, $R_{\mathrm{p}}$, is given by

$$
R_{\mathrm{p}}=-\frac{\mathrm{d}[\mathrm{M}]}{\mathrm{d} t}=k_{\mathrm{p}} \times\left[\mathrm{P}^{*}\right]_{0} \times[\mathrm{M}]
$$

where $k_{\mathrm{p}}$ is the rate constant of propagation, $\left[\mathrm{P}^{*}\right]_{0}$ is the initial concentration of living chains (= constant), and $[\mathrm{M}]$ is the monomer concentration. This leads to conventional first-order dependence

$$
\ln \frac{[\mathrm{M}]_{0}}{[\mathrm{M}]_{t}}=k_{\mathrm{p}} \times\left[\mathrm{P}^{*}\right]_{0} \times t=k_{\mathrm{app}} \times t
$$

For the polymerization in the presence of aluminium alkyls, the apparent rate constant of propagation, $k_{\text {app }}$, decreases during polymerization, ${ }^{1-3}$ i.e., we have to take into account the existence of two (or more) active species with different reactivities or a decrease of the concentration of living chains. Compared to polymer chains in solution, those incorporated into the network

\section{initiator}

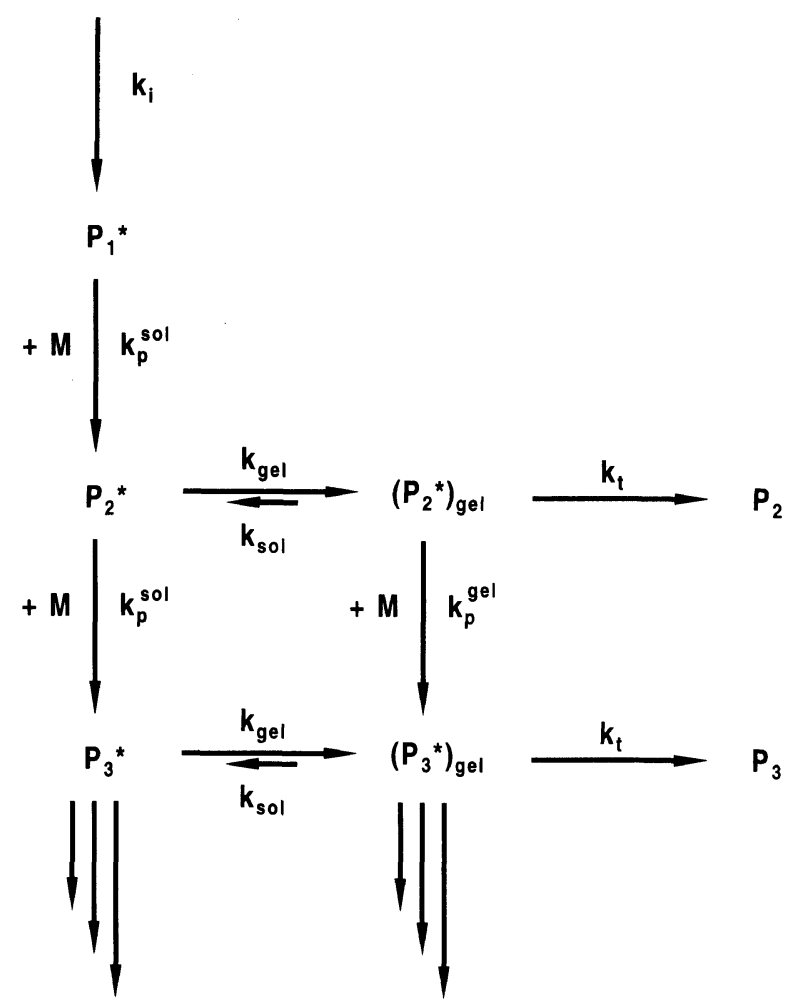

Scheme 1. Kinetic scheme of the anionic polymerization in the presence of aluminium alkyls. Termination reactions are assumed to result exclusively from the incorporation of living chains into the network (see above).

are considerably less reactive because the co-ordinated aluminate end group has a lower nucleophilicity ${ }^{2}$ and is more bulky ( $c f$., Figure 4 ). In addition, due to the precipitation of the network, the accessibility of the living chain ends towards monomer in a swollen polymer gel is decreased. Thus, we can develop the following kinetic scheme (Scheme 1):

The apparent rate constant of propagation is then given by

$$
k_{\mathrm{app}}=k_{\mathrm{p}}^{\mathrm{sol}} \times\left[\mathrm{P}^{*}\right]^{\mathrm{sol}}+k_{\mathrm{p}}^{\mathrm{gel}} \times\left[\mathrm{P}^{*}\right]^{\mathrm{gel}}
$$

where $k_{\mathrm{p}}^{\mathrm{sol}}, k_{\mathrm{p}}^{\mathrm{gel}},\left[\mathrm{P}^{*}\right]^{\text {sol }}$, and $\left[\mathrm{P}^{*}\right]^{\text {gel }}$ are the rate constants of propagation and the concentrations of active chains in solution and in the network, respectively. If we assume that the reactivity of the polymer chains is such low that we can regard them as 'dormant' chains, i.e., $k_{\mathrm{p}}=$ $k_{\mathrm{p}}^{\mathrm{sol}} \gg k_{\mathrm{p}}^{\mathrm{gel}} \approx 0$, the concentration of active chains decreases as the network precipitates from the reaction solution. Thus, eq 3 can be transformed to

$$
k_{\mathrm{app}, t}=k_{\mathrm{p}} \times\left[\mathrm{P}^{*}\right]_{t}^{\mathrm{sol}}=k_{\mathrm{p}} \times \alpha \times\left[\mathrm{P}^{*}\right]_{0}=\alpha \times k_{\mathrm{app}, 0}
$$

Thus, the fraction of active chain ends, $\alpha$, is given as

$$
\alpha=\frac{k_{\mathrm{app}, t}}{k_{\mathrm{app}, 0}}
$$

and can be calculated from the ratio of the slope of the first-order time-conversion plot at a time $t$ and the initial slope ( $c f$., Figure 7 and Table 1). In the absence of termination reactions, the value of $\alpha$ should be equal to the mole fraction of soluble polymer, $x_{\text {sol }}$, which is determined from the weight fraction, $w_{\text {sol }}$, and the corresponding molecular weight, $M_{n, \text { sol }}$. 
Table I. Determination of the apparent rate constants and the composition of the reaction solution of the polymerization described in Figure $7^{\mathrm{a}}$

\begin{tabular}{|c|c|c|c|c|c|c|c|c|c|}
\hline$\frac{t}{\min }$ & $x_{\mathrm{p}}$ & $\frac{k_{\mathrm{app}, t}}{\min ^{-1}}$ & $\alpha$ & $M_{n, \mathrm{gel}}$ & $M_{n, \mathrm{sol}}$ & $w_{\mathrm{sol}}$ & $x_{\mathrm{sol}}$ & \multicolumn{2}{|c|}{$\begin{array}{l}M_{n} \quad M_{w} / M_{n} \\
\text { (complete MWD) }\end{array}$} \\
\hline 0 & 0 & 0.250 & 1 & - & - & - & - & - & - \\
\hline 1 & 0.18 & 0.165 & 0.66 & - & - & - & - & 630 & 1.28 \\
\hline 2.5 & 0.34 & 0.089 & 0.36 & 1080 & 470 & 0.52 & 0.72 & 750 & 1.42 \\
\hline 5 & 0.42 & 0.045 & 0.18 & 1190 & 500 & 0.24 & 0.43 & 880 & 1.54 \\
\hline 15 & 0.59 & 0.031 & 0.12 & 1830 & 530 & 0.07 & 0.21 & 1460 & 1.60 \\
\hline
\end{tabular}

${ }^{\mathrm{a}} x_{\mathrm{p}}$, monomer conversion; $k_{\mathrm{app}, t}$, apparent rate constant; $\alpha$, fraction of active chains; $w$, weight fraction; $x$, mole fraction; $M_{t}$, number-average molecular weight (GPC); $M_{w} / M_{n}$, polydispersity index.

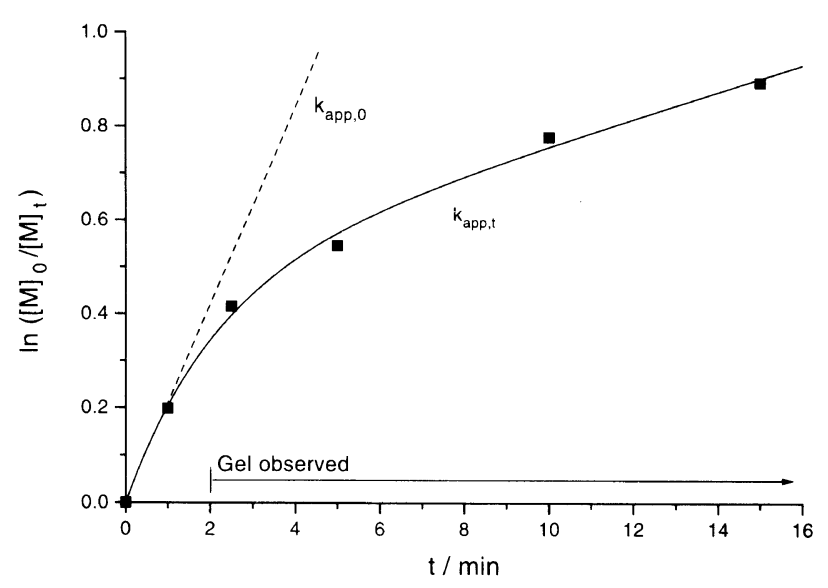

Figure 7. First-order time-conversion plot of the polymerization of MMA in toluene at room temperature: $[\mathrm{tBuLi}]_{0}=1.7 \times 10^{-2} \mathrm{moll}^{-1}$, $\left[\mathrm{AlEt}_{3}\right]=3.4 \times 10^{-2} \mathrm{moll}^{-1},[\mathrm{MMA}]_{0}=0.2 \mathrm{moll}^{-1}$. The data result from separate experiments, $\left[\mathrm{P}^{*}\right]_{0} \approx 9.35 \times 10^{-3} \mathrm{moll}^{-1}$.

Since the most significant effect on $\left[\mathrm{P}^{*}\right]_{t}$ occurs at the kink in time-conversion plot, i.e. during the very early polymerization steps, we investigated a polymerization expecting a considerably low molecular weight polymer. Here, the kink in the first-order time-conversion plot and the precipitation of the polymer gel appear simultaneously after $c a .2$ min (see Figure 7). The values of $\alpha$ and $x_{\text {sol }}$ for this polymerization are summarized in Table 1.

Figure 8 shows that the fraction of soluble chains decreases exponentially with time reaching a plateau at $x_{\text {sol }} \approx 0.21$ for $t \geq 15 \mathrm{mim}$ which may correlate to the thermodynamic equilibrium between chains in solution and in gel. A similar decay is determined from the polymerization kinetics with a limiting value of $\alpha \approx 0.12$. Thus, the network formation is seen as the predominant reason for the kink in the time-conversion plot. The fact that the fraction of soluble chains, $x_{\text {sol }}$, is higher than the fraction of active chains, $\alpha$, may indicate that the network precipitation is not exclusively responsible for the decrease in rate but also termination reactions (see above). If we, however, assume that the sol-gel equilibrium is established faster than it takes for the workup procedure, the determined fraction of chains in solution might be higher than its equilibrium value.

\section{Routes to Prevent Network Formation}

The co-ordinated polymer network is insoluble in toluene whereas it dissolves in polar or electron-donating

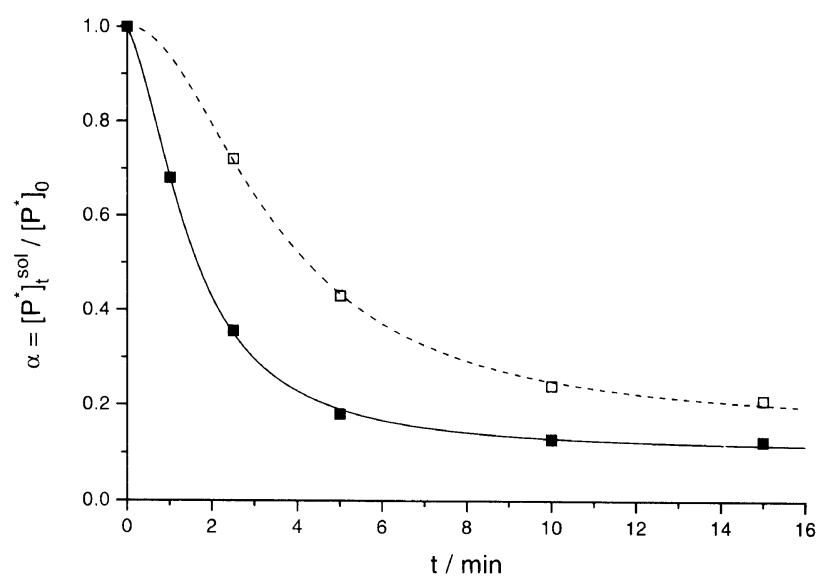

Figure 8. Plot of the fraction of active polymer chains, $\alpha$, and fraction of soluble polymer, $x_{\text {sol }}$, vs. time of the polymerization described in Figure 7. $\square, \alpha$, calculated from kinetics according to eq 5 $\left(k_{\mathrm{app}, 0}=0.25 \mathrm{~min}^{-1}\right) ; \square, x_{\mathrm{sol}}$, determined gravimetrically.

solvents like tetrahydrofuran. Considering again the structures in Figure 4, this break-up of the network can be explained by a co-ordination of tetrahydrofuran molecules instead of a living polymer chain to each aluminate end group. Apart from the aspect of a more polar reaction medium, the network formation should also be suppressed by introducing a more bulky aluminate end group.

In fact, the only examples in literature for controlled and living methacrylate polymerizations in the presence of aluminium alkyls, do all comply with the above aspects. For anionic polymerization, Ballard et al. ${ }^{11}$ used bulky aluminium phenoxides at high initial monomer concentrations in toluene whereas Teyssié et al. ${ }^{12}$ obtained the best results when polymerizing in polar pyridine solution. On the other hand, Inoue et al. ${ }^{13}$ introduced a porphyrin ligand to the living chain end, thus combining in a very elegant way electron-donating and steric aspects.

Acknowledgments. We wish to thank Dr. Katharina Landfester (Max-Planck Institut für Polymerforschung, Mainz) for performing the CP-MAS solid-state NMR measurements and Peter Blumers for his very helping hands. This work was supported by the Bundesministerium für Bildung, Wissenschaft, Forschung und Technologie and BASF AG, Ludwigshafen (Project No. 03N 3006 A5). 


\section{REFERENCES}

1. H. Schlaad and A. H. E. Müller, Macromol. Symp., 95, 13 (1995).

2. H. Schlaad and A. H. E. Müller, Macromol. Rapid Commun., 16, 399 (1995).

3. H. Schlaad and A. H. E. Müller, Macromol. Symp., 107, 163 (1996).

4. P. Hubert, A. Soum, and M. Fontanille, Makromol. Chem., Macromol. Symp., 85, 313 (1994).

5. K. Hatada, private communication.

6. K. H. Hunt, M. C. Crossman, D. M. Haddleton, P. M. Lloyd, and P. J. Derrick, Macromol. Chem., Rapid Commun., 16, 725
(1995).

7. H. Schlaad, H. Kolshorn, and A. H. E. Müller, Macromol. Rapid Commun., 15, 517 (1994).

8. Y. Takashi, Bull. Chem. Soc. Jpn., 40, 612 (1967).

9. R. Benn, A. Rufinska, H. Lehmkuhl, E. Janssen, and C. Krüger, Angew. Chem., 95, 808 (1983).

10. H. Weiß, H. Schlaad, and A. H. E. Müller, unpublished results.

11. D. G. H. Ballard, R. J. Bowles, D. M. Haddleton, S. N. Richards, R. Sellens, and D. L. Twose, Macromolecules, 25, 5907 (1992).

12. J.-S. Wang, R. Jérôme, and P. Teyssié, Macromolecules, 28, 2990 (1995).

13. T. Adachi, H. Sugimoto, T. Aida, and S. Inoue, Macromolecules, 26, 1238 (1993). 